\title{
La sociología sin método: la raíz hegeliana del pensamiento de Luhmann
}

\author{
SOCIOLOgY WITHOUT METHOD: THE HEgELIAN ROOT OF LUHMANN'S THINKING
}

Mauricio Casanova (m.casanovabrito@gmail.com) Instituto de Estudios Latinoamericanos, Universidad Libre de Berlín (Berlín, Alemania)

\begin{abstract}
Luhmann's theory has been commonly considered as a radical overcoming of the traditional philosophy. The interpreters often refer to the non-ontological background of the theory as the criticism of the conscience's centrality, the emphasis in conflict and distinction and the influence of sciences as cybernetic, biology and mathematics. In the present paper we try to demonstrate that there is also an important philosophical heritage in the Luhmann's sociological work: the Hegelian heritage. We refer to four main points: the congruence of being and thought; the being as the unity of unity/distinction; the rationality as auto-reference and the congruence between theory and society. The focus of our analysis is on the complications of the system theory when it tries to eliminate the idea of spirit.
\end{abstract}

Key words: Luhmann, Hegel, system theory, dialectical philosophy, sociology.

\section{Resumen}

La teoría de Luhmann ha sido considerada comúnmente como una radical superación de la filosofía tradicional. Los interpretes se refieren frecuentemente a los antecedentes no ontológicos de esta, como la crítica a la centralidad de la conciencia, el énfasis en el conflicto y la distinción y la influencia de ciencias como la cibernética, la biología y las matemáticas. En el presente artículo se intenta demostrar que existe también otra importante herencia en la obra sociológica de Luhmann: la herencia hegeliana. Los principales puntos analizados son: la congruencia entre ser y pensamiento; el ser como unidad de unidad/distinción; la racionalidad como auto-referencia y la congruencia entre teoría y sociedad. El enfoque de nuestro análisis radica en las dificultades de la teoría de sistemas al intentar eliminar la idea de espíritu.

Palabras clave: Luhmann, Hegel, teoría de sistemas, dialéctica, sociología.

\section{Introducción}

La obra Luhmann ha sido interpretada -tanto por sus adeptos como por sus detractores- de diversas maneras. Las tendencias generales son tres: las apreciaciones similares a la consideración que el mismo Luhmann tiene sobre su propio pensamiento; las interpretaciones que asocian las ideas del autor con herencias indirectas, prejuicios o aspectos ideológicos preconcebidos; las consideraciones que tienden a relacionar la obra de Luhmann con la herencia filosófica alemana o la crítica francesa al estructuralismo. 
Los autores pertenecientes a esta primera tendencia aseveran, al igual que Luhmann, que la teoría social de sistemas, el cambio de paradigma anunciado en Sistemas sociales (1991:25), se origina de la aplicación de los postulados de la teoría general de sistemas (en el contexto de la física, la biología y la cibernética) y la filosofía (fundamentalmente, la fenomenología) al campo de la sociedad. Como fundamento de la necesidad de la aplicación de los postulados de estas diferentes disciplinas, se indica frecuentemente la presencia de una crisis ocasionada por la incongruencia entre el pensamiento sociológico y la evolución de la sociedad: "pero la evolución de la sociedad no se detiene, y, aunque duela, tampoco sigue los patrones tan cuidadosamente elaborados por los sociólogos" (Rodríguez y Arnold 2007:XVII). Arnold comparte esta consideración. En referencia a las descripciones contemporáneas de la sociedad, sostiene que "estas no explican satisfactoriamente los fenómenos que describen y no van más allá de resaltar los aspectos parciales de la sociedad, pues carecen de una concepción de la misma y de su propia posición para observarla" (2007:XXXI). Rodríguez reitera, en alusión al estado de las ciencias sociales previo a la obra de Luhmann, que "hacía falta un trabajo de envergadura que, con la solidez que los clásicos tuvieron para analizar la sociedad en que les correspondió vivir, pudiera ofrecer una teoría solvente acerca de la sociedad actual" (2007:VI). De la misma manera, Izuzquiza, asevera que: "Luhmann inicia su reflexión con la queja de que no disponemos actualmente de una teoría adecuada que describa lo que es nuestra sociedad contemporánea. Su propuesta teórica pretende llenar esta ausencia" (2008:9). Todos los autores -en concordancia con la perspectiva del mismo Luhmann- coinciden en el mismo diagnóstico: la incongruencia entre la realidad de la sociedad y la teoría sociológica. El argumento que fundamenta la aplicación de la teoría general de sistemas en las ciencias sociales no es teórico, sino histórico. Volveremos a analizar este punto.

Los autores pertenecientes a la segunda tendencia frecuentemente adhieren a la crítica de Habermas al funcionalismo sistémico. Esta crítica se sustenta en los siguientes aspectos: a nivel teórico, la idea de que el componente ilocucionario -asociado al significado del enunciado que produce un efecto en el mundodel acto de habla remite a un trasfondo aproblemático de la comunicación y el pensamiento (el mundo de la vida) que permanece en un modo de semi-trascendencia (la comunicación como medio descrita por Luhmann se refiere solamente a un aspecto funcional del lenguaje, no a su naturaleza); a nivel histórico (Habermas 2003), la premisa de que la sociedad moderna se caracteriza por un proceso dual y conflictivo entre, por un lado, la diferenciación interna del mundo de la vida (en sociedad, cultura y personalidad) y, por otro, la diferenciación entre el mundo de la vida y los sistemas sociales (la diferenciación funcional de la teoría de Luhmann es solamente un aspecto del proceso histórico de la modernidad); a nivel éticopolítico (Habermas 2000, 2000a), la idea de una ética -universalista, procedimental, cognitivista- del discurso destinada a formalizar las reglas racionales del argumento práctico (lo que cierra la posibilidad a una ética auto-referencial como la de Luhmann). En base a estas tres perspectivas, Habermas caracteriza la teoría social de sistemas como metabiología o pensamiento tecnificado (1993:334-453). Una crítica similar a esta -arraigada también en las ideas de Giddens- es posible encontrar en la réplica de Larraín a Mascareño (2007).

Los autores (adeptos y adeptos parciales) que tienden a relacionar la obra de Luhmann con la de otros pensadores destacan usualmente la des-trascendentalización del sujeto cognoscente, la crítica a la metafísica de la presencia, la apología de la contingencia o la afirmación de la diferencia por sobre la unidad. Mascareño, por ejemplo, al relacionar a Luhmann con Derrida, señala el énfasis común en la negatividad. Este paralelo le permite incorporar las ideas del pensador francés en la teoría de los medios de comunicación simbólicamente generalizados (2009). Miranda, por su parte, alude a la presencia indirecta - una afinidad electiva, utilizando el concepto de Weber- del humanismo negativo de la antropología filosófica alemana en el pensamiento de Luhmann: "el prejuicio humanista que 
inadvertidamente Luhmann rechaza es aquel que hace entrar en la teoría sociológica lo que podríamos llamar un humanismo positivo, pero ello lo hace desde otra tradición humanista: la del humanismo negativo; aquel que deriva de la antropología filosófica alemana" (2012:275). Miranda menciona, como representantes de este humanismo negativo, a Gehlen, Plessner, Marquard, Blumenberg y Schelsky.

Como es posible apreciar, en ninguno de los casos se destaca la influencia de Hegel. Solamente, en ciertas ocasiones, la comparación posee la forma de una analogía. Para Rodríguez y Arnold, por ejemplo, "el marco interpretativo desarrollado por Luhmann para la sociología ha sido comparado al que ofreciera Hegel a la filosofía. Del mismo modo que éste, se propuso describir nuestra época en toda su complejidad, entre su pasado y su futuro. Para ello, construyó un sistema de pensamiento que no tiene parangón en la historia de la sociología, del mismo modo que lo había hecho el filósofo del idealismo alemán" (2007:V). Esta aseveración, no obstante, no alude a una intención directa de Luhmann de utilizar la filosofía de Hegel para la elaboración de su teoría. Se refiere, más bien, a la importancia entre el autor y la época en la que surge su obra. En general, se tiende a considerar la obra de Luhmann no como la herencia de una doctrina filosófica, sino como una instancia de radical superación de la filosofía: "Toda la obra de Luhmann puede considerarse como una crítica contra esa "vieja filosofía europea», que debe ser tenida en cuenta para comprender muchos de los elementos de su obra" (Izuzquiza 2008:74).

En el presente trabajo argumentamos que es posible referirse a una herencia hegeliana en la obra de Luhmann, lo que se puede apreciar en cuatro puntos, que expondremos en cada caso analizando respectivamente la obra de Hegel y la re-consideración en Luhmann: la congruencia ser/pensamiento (I), el devenir del ser como unidades de unidad/diferencia que retornan a la misma unidad (II), la racionalidad como forma suprema de auto-referencia (III) y la concordancia pensamiento/época histórica (IV).

\section{Reflexionar desde adentro: la congruencia entre ser y pensar}

El pensamiento filosófico moderno, formulado generalmente en torno al modelo de las ciencias naturales, se sustentaba en los preceptos de la lógica tradicional, a saber, el principio de identidad y no contradicción, en donde el ser es idéntico a sí mismo y no es lo que no es. El enfoque era el descubrimiento de fuerzas físicas inalterables. El modelo ideal de texto científico era la Enciclopedia. Bajo la influencia de la física moderna, se afirmaba "que la historia de la filosofía es constantemente un subconjunto de la historia de la astronomía, que una gran filosofía existe siempre con una cosmología, que ésta es el índice crucial de aquella" (Enthoven 2009:203).

A fines del siglo XVIII, no obstante, en una época de fuertes alteraciones al orden tradicional, empieza a surgir otro enfoque: lo que es menester comprender es la transformación, el cambio, la historia; y no la estabilidad que trasciende y determina todos los sucesos humanos (los ciclos de auge y decadencia de los imperios) y naturales (las leyes del universo). Para Koselleck, "en estos siglos se produce una temporalización de la historia cuyo final se encuentra aquel tipo peculiar de aceleración que caracteriza a nuestros modernos" (1993:23). Para Gadamer: "La aparición de una toma de conciencia histórica es verdaderamente la revolución más importante de las que hemos experimentado tras la llegada de la época moderna. Su contenido espiritual sobrepasa probablemente aquel que reconocemos en las realizaciones de las ciencias naturales, realizaciones que tan visiblemente han trasformado la superficie de nuestro planeta. La conciencia histórica es un privilegio, quizá incluso una carga que, como tal, no ha sido impuesta de ninguna otra de las generaciones anteriores" (1993:41). 
En este contexto, los principios de la lógica tradicional resultaban inoportunos. Para confrontar este problema hubo dos posturas; en lo que refiere al tema del conocimiento histórico. Primero, el historicismo, que -considerando la imposibilidad del conocimiento histórico certero-recomendaba realizar inferencias solamente a partir de las evidencias documentales de los hechos del pasado. Segundo, la reflexión filosófica sobre el devenir humano en el tiempo, en la que destacan dos tendencias: la idea, constante en el siglo XVIII, de que la historia universal corresponde al continuo progreso de las facultades naturales innatas del ser humano; la idea, constante en el siglo XIX, de que la raíz misma de la constitución de la naturaleza humana se encuentra en el devenir histórico: "On this approach the very nature of human beings is subject to evolution and cannot be understood independently of the exertion of human faculties in history. History is, thus, constitutive of the metaphysics of human nature" (Perinetti 2006: 1122). De esta última tendencia Hegel fue el principal representante.

Para Hegel, no era posible oponer simplemente lo no histórico con lo histórico, como sus antecesores, sino que era necesario concebir a lo finito como una instancia de la misma infinitud: "no es que la infinitud consista pues, en la supresión de la finitud en general sino que lo finito es sólo esto: por su propia naturaleza llega a ser infinitud. La infinitud es su determinación o sea aquello que él es en sí" (2011:262). Hasta el mismo conocimiento filosófico debía ser considerado como instancia de este devenir; no como la organización de las condiciones atemporales del pensamiento (Kant) ni como la iluminación de substancias ideales (Platón). El conocimiento, en tanto episodio situado históricamente, opera como escena particular de una trama general: el devenir ser del espíritu. No es que este se descubra en el pensamiento, sino que el pensamiento es una instancia del desarrollo del espíritu. Sujeto y objeto coinciden. Ser y no-ser conviven. Identidad y diferencia poseen una unidad común. Por esta razón, a pesar de lo que Marx consideraba en La miseria de la filosofía, el método hegeliano no consiste en la tradicional triada tesisantítesis-síntesis: no hay método hegeliano como no hay moral o política hegeliana. Existe la filosofía desarrollada en el tiempo y que Hegel intenta interpretar. Para esto, es menester reflexionar desde dentro. El método es el movimiento mismo de la realidad captado conceptualmente: "el método no es, en efecto, sino la estructura del todo, presentada en su esencialidad pura" (1966:32). Al igual que en la realidad, el conocimiento del sí mismo -el concepto- no manifiesta su verdad sino incluyendo al no ser, al no concepto: "lo verdadero es el todo" (1966:16). El desarrollo de la filosofía consiste "en no hallarse separado de su contenido y, de otra, en determinar su ritmo por sí mismo" (1966:38). Mediante el pensamiento filosófico, el espíritu señala "la naturaleza interior de su propio destino" (1955:8). De esta manera, "el espíritu que se sabe desarrollado así como espíritu es la ciencia. Ésta es la realidad de ese espíritu y el reino que el espíritu se construye en su propio elemento" (1966:19).

A esta postura -planteada inicialmente por Fichte en su crítica a Kant y reconsiderada posteriormente por Hegel (abandonando la primacía del sujeto) - que entiende al pensamiento como devenir del ser, se le denomina dialéctica: "in clarifying the method for articulating basic transcendental relations, Fichte differentiated himself from Kant by (1) generalizing the model of self-world corollaries, and (2) reconstructing and deducing these relations by means of a new method, the first method that can be described as dialectical" (Henrich 2002:24). Considerando esta postura -el pensamiento como instante en la realización del ser-el argumento de Hegel para el inicio del pensamiento filosófico no podía haber sido otro que el cambio en la realidad, en el objeto, en el devenir de los asuntos humanos, y la consecuente incongruencia con el pensamiento hasta entonces disponible: "No es difícil darse cuenta, por lo demás, de que vivimos en tiempos de gestación y de transición hacía una nueva época. El espíritu ha roto con el mundo anterior de su ser allí y de su representación y se dispone a hundir eso en el pasado, entregándose a la tarea de su propia transformación. El espíritu, ciertamente, no permanece nunca quieto, sino que se halla siempre en movimiento incesantemente progresivo. Pero, así como en el niño, tras un largo periodo 
de silenciosa nutrición, el primer aliento rompe bruscamente la gradualidad del proceso puramente acumulativo en un salto cualitativo, y el niño nace, así también el espíritu que se forma va madurando lenta y silenciosamente hacía la nueva figura, va desprendiéndose de una partícula tras otra de la estructura de su mundo anterior y los estremecimientos de este mundo se anuncian solamente por medio de síntomas aislados; la frivolidad y el tedio que se apoderan de lo existente y el vago presentimiento de lo desconocido son los signos premonitorios de que algo otro se avecina. Estos paulatinos desprendimientos, que no alteran la fisonomía del todo, se ven bruscamente interrumpidos por la aurora que de pronto ilumina como un rayo la imagen del mundo nuevo" (Hegel 1966:12).

En la dialéctica, primero es la realidad de la historia, el largo periodo de silenciosa nutrición, luego el pensamiento, la aurora que de pronto ilumina como un rayo la imagen del mundo nuevo. Para el marxismo: primero el capitalismo y sus contradicciones, luego la revolución socialista.

Desde entonces, esta postura y el trascendentalismo kantiano -los inicios del pensamiento como a priori (Kant) o como resultado (Hegel)- han convivido en constante conflicto. Hegel, optando por el camino de la dialéctica, se refiere a ambas posturas de la siguiente manera: "Aunque la filosofía, como el pensamiento y la comprensión del espíritu de una época, es necesariamente algo apriorístico [el énfasis es nuestro], es también al mismo tiempo y no menos esencialmente, un resultado, puesto que el pensamiento se produce, más aun, es la vida y la actividad que consisten en producirse. Esta actividad encierra el momento esencial de una negación, en cuanto que producir es, al mismo tiempo, aniquilar, pues la filosofía, para poder producirse, tiene como punto de partida lo natural, de lo que arranca para superarlo" (1955:53).

Como sostuve en otra instancia, ambas propuestas fueron representadas a fines del siglo $\mathrm{XX}$, en el contexto de las ciencias sociales, por la pragmática trascendental de Habermas y la teoría social de sistemas de Luhmann: "en la controversia entre la teoría crítica de Jürgen Habermas y el funcionalismo sistémico de Niklas Luhmann las ideas de Kant y Hegel (las posibilidades de iniciar el pensamiento filosófico y teórico como algo apriorístico o como un resultado) vuelven a confrontarse, pero no en términos de filosofía de la historia sino de teoría de la sociedad" (Casanova 2014:95).

El argumento de Luhmann para el inicio del pensamiento sociológico es similar al de Hegel para la filosofía. También se alude a la discordancia entre la realidad -en este caso, de la sociedad- y el pensamiento. Ya no es posible pensar en una sociedad centrada en la moral, en una elite social o en un criterio racional universal debido a que en la sociedad misma ya no existe un centro. Este punto es crucial: no dice Luhmann que no haya existido nunca, sino que dejó de existir. "El desiderátum metodológico de la comparación funcional refleja peculiaridades de la sociedad moderna [la cursiva es nuestra]; allí se encuentra una razón más para ya no confiar en lo que ofrece la tradición. Porque la sociedad moderna -cosa que demostraremos ampliamente- se caracteriza por la autonomización funcional y la clausura operativa de sus sistemas parciales más importantes" (2007:26). Cambia la sociedad, el pensamiento ya no concuerda con esta, surge la teoría social de sistemas. El mismo reclamo de Hegel hacia la filosofía que separaba lo finito de lo infinito, lo subjetivo de lo objetivo, lo material de lo ideal -“se dice, por ejemplo, que el sujeto es distinto del objeto, o también que lo finito es distinto de lo infinito, etc. y rechazan entonces la idea especulativa como si la unidad espiritual concreta fuese en sí misma carente de determinación y no contuviera dentro de sí la distinción" (2005:65) - reitera Luhmann en referencia a la centralidad del sujeto en las ciencias sociales. En ambos casos se acusa al adversario de tradición, atraso, ceguera, y se remarca en la necesidad de entender al pensamiento como parte del devenir de lo descrito. Esta es la apuesta teórica central de Luhmann: "Las siguientes investigaciones se ocuparan del sistema social llamado 
sociedad moderna. Un intento de tales características -y esto es de lo que hay que rendir cuentas primeroactualiza una relación circular con su objeto" (2007:5).

Tanto en Hegel como en Luhmann es menester reflexionar desde dentro. La diferencia es que Hegel se refiere al espíritu y Luhmann a la sociedad: "El intento por describir a la sociedad no puede hacerse fuera de la sociedad: hace uso de la comunicación, activa relaciones sociales y se expone a la observación en la sociedad. Entonces, como quiera que pretenda definirse el objeto, la definición misma es ya una de las operaciones del objeto: al realizar lo descrito, la descripción se describe también a sí misma" (Luhmann 2007:5). En ambos casos, el inicio del pensamiento como a priori (Kant, Habermas), como la formulación de condiciones incondicionadas del argumento teórico y práctico, es desechado. El pensamiento opera como resultado del devenir de la sociedad y no como la generalización de la posibilidad de pensar o de comunicarnos en la sociedad: "Podemos siempre preguntarnos por las condiciones físicas, lingüísticas y sociológicas del conocimiento; pero la investigación que se lleve a cabo en torno a ello debe ser siempre autológica, es decir, debe tener en cuenta las consecuencias que de allí se desprendan para nuestra propia actividad" (Luhmann 1996:10). Para Luhmann, "las teorías trascendentales obstruyen cualquier inferencia autológica que recaiga en sí mismas" (2007:15). Para Baecker: "there are no longer any transcendental resources to social systems in Luhmann's theory of them. There is no need to develop a whole concept of interpenetration to make sure that interchange between not only the normative and the interactive but also between the personality and the behavioral or social and the personal system takes place" (2014:23).

Uno de los principales postulados de la teoría social de sistemas consiste en la afirmación de que el argumento dialéctico - que el conocimiento se considere a sí mismo como autorrealización de lo descritono puede reducirse al campo de la conciencia subjetiva: "Es usual hablar de conocimiento y ciencia sirviéndose de una conceptualidad referida al sujeto. De acuerdo con ello, el sujeto del conocimiento es el hombre o, en todo caso, la conciencia del hombre, o posiblemente el peculiar colectivo de la conciencia trascendental del ser humano" (Luhmann 1996:13). Para Luhmann, el tránsito de la conciencia a la autoconciencia mediante unidades de diferencia que retornan a la misma unidad es también una propiedad de la comunicación. Esto impulsa al autor a secularizar el pensamiento dialéctico: abandonar la idea de la racionalidad como instancia en la autorrealización del todo -el espíritu- y a considerar a la sociedad moderna como la proliferación (los sistemas sociales) de distintos universos internos de racionalidad entendida esta como el nivel supremo de auto-referencia- cada cual con pretensiones de universalidad. En los próximos apartados analizaremos esta propuesta implícita de superación del pensamiento dialéctico, enfatizando en las dificultades de Luhmann al intentar erradicar la idea hegeliana de espíritu.

\section{El ser como unidad de unidad y diferencia}

Para Hegel, la filosofía no puede reducirse -como el pensamiento formulado al amparo de la ciencia moderna- a la dilucidación de las condiciones incondicionadas (trascendentales) del conocimiento; como en Kant, para el que la ciencia "se reduce a su esquema inerte, a un esquema propiamente dicho, haciendo descender la organización científica a un simple diagrama" (Hegel 1966:33). El pensamiento no posee una estructura interna estable, no obedece a leyes o parámetros, sino que constituye una totalidad que se da ella misma a sí misma: "el representar tiene por contenido esa materia sensible, pero bajo la determinación de lo mío, o sea de que tal contenido está en mí" (Hegel 2005:128). En la lógica es posible apreciar que "el pensamiento y lo universal son precisamente eso: que él es él mismo y su otro, que alcanza de éste y nada se le escapa" (Hegel 2005:129). En el trascendentalismo, se intenta formular los supuestos a priori que hacen posible el pensamiento metafísico: "Kant thought that he had devised a way of asking first about the very possibility of any philosophical or a priori knowledge, or even of any experiential 
knowledge, a way of determining the conditions necessary to be able to say at all how things are, whether a posteriori or a priori" (Pippin 2012:20). En la dialéctica de Hegel, en cambio, las leyes del pensamiento y la lógica son las leyes de las cosas mismas, el ser (en constante cambio) se representa a sí mismo en el concepto: "Al intentar el pensamiento hacerse con un concepto de las cosas, éste (y con él juntamente, de sus formas más inmediatas, juicio y silogismo) no puede consistir en determinaciones y relaciones que sean extrañas y exteriores a la cosa [...] Esta última expresión es, sin embargo, incómoda porque demasiado comúnmente se considera que el pensamiento sólo pertenece al espíritu o a la conciencia y lo objetivo se usa a su vez y en primer lugar para lo no espiritual” (Hegel 2005:131).

En la crítica de Hegel a Kant: "Reason can still be said to "have insight only into what it itself produces according to its own design", but reflective attention to what it so "produces», what reason requires of nature and spirit, reveals (a) an essentially temporal or developmental structure to such requirements and (b) that such "producings» were not to be thought of as «imposed» on some exogenous material of experience, and so were not subject to Kant's critical restrictions about the unknowability of things in themselves" (Pippin 2012:24).

En la lógica hegeliana, por ende, las partes no reflejan particularidades o generalidades de la estructura interna del pensamiento, sino instancias en el devenir del espíritu en el tiempo. En la primera parte, la doctrina del ser, "el puro ser constituye el comienzo porque es tanto pensamiento puro como lo inmediato simple e indeterminado, y el primer comienzo no puede ser nada mediato ni más determinado" (Hegel 2005:125). De la unidad del ser y la nada (el ser es igual sí mismo y no es lo que no es, la nada es igual a sí misma en su no ser el ser) surge el devenir, la existencia: "El ser en el devenir, en cuanto uno con la nada, así como la nada en cuanto una con el ser, son solamente desapareciendo; el devenir coincide, por su contradicción interna, con la unidad en la cual ambos están superados; su resultado es, por consiguiente, el existir" (Hegel 2005:194). El ser, en su primera instancia, es ya distinción.

La segunda parte, la doctrina de la esencia, trata sobre el "ser o la inmediatez, que mediante la negación de sí mismo, es mediación consigo y referencia a sí mismo, y es así igualmente mediación que supera en referencia a sí o inmediatez: la esencia" (Hegel 2005:207). Lo positivo (la carencia de oposición) y lo negativo (la distinción referente a otras identidades) forman una unidad: "La distinción en sí es la distinción esencial, lo positivo y lo negativo, y eso de tal manera que lo positivo es así la idéntica referencia a sí que no es lo negativo, y éste por su parte es de tal modo lo distinto de por sí que no es lo positivo. Siendo así cada uno de ellos algo de que por sí que no es lo otro, cada uno aparece en lo otro y sólo es en tanto lo otro también es" (Hegel 2005:216). Es importante, para el problema de esta investigación, destacar en este punto que para Hegel el universo se compone por una multiplicidad de esencias (conformadas por la unidad de la diferencia entre la existencia y su oposición) en donde cada cual es el otro lado de la distinción para las otras esencias: "La existencia es la unidad inmediata de la reflexión hacia sí y de la reflexión hacia otro. Es, por consiguiente, la multitud indeterminada de existentes en tanto reflejada hacia sí que a la vez igualmente aparecen vueltos hacia otro, son relativos y forman un mundo de dependencia mutua y de una conexión infinita de fundamentos y fundada. Los fundamentos son ellos mismos existencias, y los existentes igualmente, son tanto fundamentos hacia muchos como fundados" (Hegel 2005:220).

No es extraño remitir al paradigma sistema/entorno al leer estas palabras de Hegel. Un argumento similar - unidades de unidad/diferencia que retornan a la misma unidad, o la identidad de identidad/diferencia que regresa a la misma identidad- utiliza Hegel en referencia al tránsito desde la conciencia inmediata a la autoconciencia: las fases de la lógica remiten a fases en el devenir temporal del pensamiento. 
La conciencia surge con la certeza sensible inmediata: la aprehensión momentánea -que no distingue verdad o falsedad- del objeto, en donde tanto el sujeto de la certeza como el objeto aprehendido son solamente debido a que son. La conciencia "es yo y nada más, un puro éste; el singular sabe un puro esto o lo singular" (Hegel 1966:63). El yo "no significa un representarse o un pensar múltiple, ni la cosa tiene la significación de múltiples cualidades, sino que la cosa es, y es solamente porque es" (Hegel 1966:63). La aprehensión de la cosa no se debe a que el sujeto se ha desarrollado como pensar o porque el objeto tenga un comportamiento diverso con respecto a otras cosas: "yo tengo la certeza por medio de otro, que es precisamente la cosa; y éste, a su vez, es en la certeza por medio de otro, que es precisamente el yo" (Hegel 1966:64). La forma de la verdad es, por ende, la de un universal: "al decir este aquí, este ahora, este singular, digo todos los estos, los aquí, los ahora, los singulares; y lo mismo, al decir yo digo este yo singular, digo en general, todos los yo; cada uno de ellos es lo que digo: yo, este, yo singular" (Hegel 1966:66). La conciencia se sabe a sí misma como sujeto único, pero lo afirmado es posible de enunciar en todos los sujetos: es un ego universal, que es en la medida en que no es todo lo demás: "a este algo simple, que es por medio de la negación, que no es esto ni aquello, un no esto al que es también indiferente el ser esto o aquello, lo llamamos universal; lo universal es, pues, lo verdadero de la naturaleza sensible" (Hegel 1966:65). La conciencia no es esencia, en el sentido de la lógica, pues no supera la fase -en cada casofugaz del constante haber-sido: "su verdad consiste, sin embargo, en haber sido. Pero lo que ha sido no es, de hecho, una esencia; no es, y de lo que se trata es de ser" (Hegel 1966:71). Hegel no inicia entonces el análisis del pensar mediante la relación interioridad/exterioridad, sensibilidad y cosa en sí, como en Kant, sino con la distinción misma desplegada por la conciencia inmediata: el inicio del teatro del pensamiento dirigido hacia lo absoluto.

Para el conocimiento filosófico, sostiene Hegel, la certeza sensible aparece como proceso dialéctico en el que ambas partes, el yo y la cosa en su inmediatez, forman el proceso de percepción. El universal se particulariza como objetivo percibido y sujeto perceptor. La cosa, en este caso, opera como médium de las cualidades indeterminadas: "ahora es un médium común universal en el que muchas propiedades, como universalidades sensibles, son cada una para sí, y como determinadas, excluyen a las otras" (Hegel 1966:74). Con esto, es posible que cada cualidad aparezca de manera diferente para cada cual: un objeto es de un color para mi vista, de un sabor para mi gusto, de una forma para mi tacto. Desaparece, por tanto, la certeza inmediata: aparecen la duda y el error.

El entendimiento, la verdad como universal no condicionado por los sentidos, surge del sujeto frustrado por la complejidad de las diversas percepciones subjetivas. En este, las relaciones entre los objetos se muestran como fuerzas: "diferencias establecidas como independientes [que] pasan de modo inmediato a su unidad; [la que] pasa a ser también de modo inmediato el despliegue, y el despliegue retorna, a su vez, a la reducción" (Hegel 1966:84). Las fuerzas, analizadas por Hegel en términos de lógicas de pensamiento, pueden ser aprehendidas como leyes, sustancia-accidente, causalidad o conciencia del mundo como mundo. En este devenir, el entendimiento aparenta inicialmente descubrir lo incondicionado que rige el movimiento de las cosas mismas -"el entendimiento supone haber descubierto aquí una ley universal que expresa la realidad universal como tal" (Hegel 1966:93)- pero lo realmente descubierto es el funcionamiento del entendimiento mismo: "el entendimiento supera las diferencias presentes en la ley, ya convertidas en diferencias puras, pero todavía indiferentes, y las pone en una unidad, que es la fuerza" (Hegel 1966:102). En este proceder, la conciencia deviene en autoconciencia.

Para la autoconciencia, la certeza es ella misma su objeto: "es igual a su verdad, pues la certeza es ella misma su objeto y la conciencia es ella misma lo verdadero" (Hegel 1966:107). La verdad se manifiesta como autorreferencia, no como lo universal, como en la certeza sensible. La percepción y el entendimiento 
aparecen como momentos -esencias que tienden a desaparecer-en el devenir temporal desde la certeza sensible a la autoconciencia: "La universalidad de la percepción, al igual que el interior vacío del entendimiento, no son ya como esencias, sino como momentos de la autoconciencia, es decir, como abstracciones o diferencias que para la conciencia son ellas mismas, al mismo tiempo, nulas o no son tales diferencias, sino esencias que tienden puramente a desaparecer" (Hegel 1966:107). La autoconciencia, en tanto nueva distinción posterior a la plasmación de la figura que reingresa a la figura misma, constituye vida: la primera manifestación del espíritu.

Al comienzo, la autoconciencia surge como deseo -apetencia egoísta- de vida, en la que lo otro se absorbe en el sujeto, se lo anula (como en la alimentación, por ejemplo). Pero, por la repetición imperecedera, este deseo abandona su carácter de autorreferencia. Lo verdadero de la autoconciencia, el despliegue del proceso dialéctico, se manifiesta en la relación entre autoconciencias, en la que la autosatisfacción excede el nivel de los instintos básicos. Lo que se desea es el reconocimiento de las otras autoconciencias: al otro como elemento del sí mismo. No se necesita vida sino herramienta de vida. En este conflicto, la autoconciencia derrotada trabaja para el goce de la otra conciencia que ha logrado ser reconocida y que es capaz de gozar del consumir el resultado del trabajo ajeno. Pero en el trabajo la autoconciencia derrotada transforma las cosas, lo producido es reflejo de su propia actividad, y su conciencia, por tanto, libre. Este proceder dialéctico entre amo y esclavo en la que "vemos repetirse el proceso que se presentaba como juego de fuerzas, pero ahora en la conciencia" (Hegel 1966:114) transita por varias fases hasta constituirse como singularidad: síntesis de conciencia y autoconciencia. Ya no se huye del mundo sino que se intenta realizarse a sí mismo en el mundo en tanto pensamiento teórico (lo real es racional) como en la actividad práctica (lo racional es real). Con esto, el pensamiento deviene en razón, como veremos en el próximo apartado.

Este proceso, plasmado no en el pensamiento sino en la sociedad misma, es descrito por Hegel en la Enciclopedia en términos de espíritu subjetivo, objetivo y absoluto. En el primero surge desde el entorno natural la conciencia humana, la que en cada caso posee como objeto a la fase precedente de sí misma. La primera manifestación de la vida se desarrolla, por tanto, fuera de la naturaleza, pero al mismo tiempo contra la naturaleza. Esta es la verdad del espíritu en su fase subjetiva. El autor distingue las siguientes etapas en el desarrollo de esta fase: el sentir inmediato de sí, por parte de la conciencia, que deviene en hábito (el sentir no es sino que se posee); la conciencia inmediata en la intuición; la imaginación y el recuerdo; la mente teórica (en la que se distingue a su vez el entendimiento, el juicio y el silogismo); la mente práctica (cuyos episodios son el sentimiento práctico, los impulsos y el reconocimiento del deberser como universal); la mente libre (síntesis de la mentalidad en sus fases teórica y práctica) en la que el ser se reconcilia con el deber-ser en la comunidad social (Hegel 2005:439-521).

En la segunda fase, el espíritu objetivo, la conciencia libre elude la finitud que le es inherente en el derecho, la moralidad y la vida ética. El primero se plasma -sucesivamente- como propiedad privada de una comunidad de individuos, como contrato de intercambio entre individuos y como legislación en contra del conflicto entre voluntades. En el mundo de la moral el sujeto se da a sí mismo la ley moral, como en Kant; su labor no queda reducida -como en el instante anterior- a respetar el derecho de otros que también reclama para sí. El deber es moral y no legal. En la vida ética surge el impulso de conquistar la libertad objetiva concreta en las instituciones sociales: la familia, la sociedad y el Estado. El Estado, en tanto manifestación suprema de lo social, es el sujeto ético auténtico. La auto-conciencia estatal es la autoconciencia de la voluntad del ciudadano (Hegel 2005:522-579). 
En el espíritu absoluto -síntesis de las fases subjetiva y objetiva- las etapas son teóricas: arte (sensibilidad), religión (representación) y filosofía (racionalidad). Esta última es concebida no tanto como en la imagen platónica del rey filósofo sino más bien como en la idea de vida contemplativa de Aristóteles. La finalidad del pensamiento filosófico es acrecentar la auto-conciencia del hombre (Hegel 2005:580-606), no indicar los caminos de su auto-realización; como propusieron luego hegelianos de izquierda o marxistas, no sin dificultades.

Uno de los principales argumentos del pensamiento de Luhmann, el cambio de paradigma que promueve para las ciencias sociales, consiste en erradicar el supuesto de que todos los procesos anteriormente descritos se ubican solamente en el escenario de la conciencia: "de acuerdo con una importante tradición, la autorreferencia se reserva para la conciencia de los sujetos, de manera que estos son interpretados como individuos que se individualizan a sí mismos. Según esto, la autorreferencia se presenta exclusivamente en el campo de la conciencia" (Luhmann 1991:390). Para Luhmann, en cambio, el proceder desde la conciencia hasta la auto-conciencia debe aplicarse tanto al campo del pensamiento como al de la comunicación: "todavía se puede decir, si se quiere salvar la terminología de sujeto, que la conciencia es el sujeto del mundo, al lado del cual existen otros tipos de sujetos, sobre todos los sistemas sociales, que los sistemas psíquicos y sociales son los sujetos del mundo" (Luhmann 1991:391). El procedimiento dialéctico ya no se concibe entonces como el movimiento de un único espíritu en la filosofía, sino que se diversifica en distintas instancias, de las cuales la ciencia -en tanto sistema social- participa pero no como centro, sino como observador observado. En este giro lingüístico de la dialéctica hegeliana, es primordial abandonar la idea de la existencia de un centro desde el cual juzgar o transformar la sociedad. Esta posibilidad es propia de un contexto en donde el devenir dialéctico del pensamiento refleja el movimiento del todo que solamente es observado por sí mismo. En palabras de Lyotard (2006), en el contexto en donde el relato de la conciencia se fundamenta en el metarrelato del espíritu. El funcionalismo sistémico se postula como un intento de dialéctica secularizada que abandona la posibilidad de un espíritu como causa primera y última de los sucesos humanos: "Podría seguirse pensando en Hegel -el único intento hasta ahora decididamente reflexionado. Pero, entonces, no se debería colocar un término como el de espíritu al final de la historia y no se debería ver en ello una idea conclusiva ni una figura de superioridad; además de que debería evitarse (contra Hegel y con Darwin) todo uso de expresiones como las de inferior o superior" (Luhmann 2007:904).

En este intento de cambio de paradigma, lo que en Hegel es la triada conciencia-autoconsciencia-razón o espíritu subjetivo-objetivo-absoluto, en Luhmann aparece plasmado como auto-referencia basal, reflexividad y reflexión. En este caso, ya no se habla de la conciencia o del espíritu, sino de la comunicación. No es pensamiento filosófico sino teoría de la sociedad. En ambos casos, no obstante, el argumento es similar: unidades que se constituyen en la medida en que la distinción unidad/diferencia que les es constitutiva, retorna a la unidad misma como conciencia de sí. Pero en Luhmann, como ya que no se trata del desarrollo de una única unidad -el espíritu- sino de la relación entre varias unidades -en un contexto de diferenciación funcional- en donde cada cual es entorno para cada cual, es necesario representar la dialéctica de una manera diferente: como diferencia, y no como unidad, de la distinción unidad/diferencia.

En la auto-referencia basal el elemento simple de la comunicación se forma de la unidad entre el sí mismo del elemento comunicativo (el ego) y lo no comunicado (que permanece en cada caso como horizonte de sentido, como posibilidad de comunicarse): "la autorreferencia basal de un sí mismo que se refiere a sí mismo es, un elemento, por ejemplo, un acontecimiento; en el caso de los sistemas sociales, una comunicación" (Luhmann 1991:395). De la misma manera que en la certeza sensible de la Fenomenología, el acontecimiento comunicativo en Luhmann sucede solamente cuando sucede. Para Hegel: "la conciencia 
natural llega, por ello, siempre, por sí misma, a este resultado, lo que en ella es lo verdadero y hace experiencia de ello; pero en seguida vuelve a olvidarlo y reinicia el movimiento desde el principio" (1966:69). Para Luhmann: "Ios acontecimientos desaparecen al generarse; en el siguiente instante no están ya en disposición para una repercusión" (1991:400). En ambas instancias, en la conciencia hegeliana y en la auto-referencia basal según Luhmann, la referencia es a otro que no es el sí mismo. En el caso de la conciencia, la referencia es a la cosa, ya sea como aprehensión instantánea, objeto de la percepción o pensamiento bajo las categorías del entendimiento. En el caso de la comunicación, la unidad de lo comunicado solamente es enunciada en referencia al universo de lo no comunicado, al horizonte indeterminado de sentido al que la comunicación puede remitir.

La reflexividad consiste en la unidad del elemento comunicativo de la auto-referencia basal (que, a su vez, es ya la unidad de una distinción) que retorna a sí misma. Es comunicación sobre comunicación: "la reflexividad recurre, entonces, a una formación de unidad que reúne una pluralidad de elementos (frecuentemente innumerables) de la cual la autorreferencia se considera parte" (Luhmann 1991:395). Así, se puede "comunicar por qué algo no fue dicho, reconocer la falsedad, gozar el dolor, gastar o no gastar dinero, comprobar el amor mediante odio y celos, decidir a no decidir, evitar el uso del poder con base en el poder" (Luhmann 1991:402). Considerando la obra de Hegel, la reflexividad corresponde a un fenómeno de autoconsciencia, pues en ambos casos la certeza es ella misma su objeto. En la autoconsciencia, como hemos visto, el amo se refiere al otro como parte constitutiva de sí mismo, mientras que el esclavo se refiere a sí mismo mediante el trabajo. En la reflexividad, considerando el contexto de diferenciación funcional de la sociedad moderna, cada comunicación sobre comunicación se refiere a lo otro (el entorno necesario para la constitución de la unidad) como parte de sí mismo, como herramienta de vida, y a sí mismo por medio de su propio trabajo (el sí mismo que en cada caso no es su propio entorno). La dialéctica señor-esclavo ya no se entiende como el devenir interno de un único espíritu, en donde el conflicto corresponde a dos autoconsciencias, sino que en cada operación de reflexividad la comunicación es señor con respecto al entorno y siervo con respecto a sí misma. En este punto, la auto-referencia de la teoría de sistemas y el trabajo de la dialéctica (ya sea en Hegel o Marx) coinciden. Este plano, poco abordado por los adherentes a la teoría social de sistemas, es, a nuestro juicio, la clave al momento de relacionar la obra de Marx con la obra de Luhmann; si es que dicha relación es posible, deseable o necesaria.

La reflexión, por último, se refiere a la comunicación sobre la unidad de auto-referencia basal y reflexividad: es una selección (reflexividad) de una selección de una comunicación (auto-referencia basal) que se diferencia a sí misma de su entorno. La unidad del sí mismo (sistema) y lo otro (entorno) retorna a la misma unidad, se comunica sobre sí misma: "en este caso, el sí mismo es el sistema al que la operación autorreferencial se adscribe; se realiza como una operación con la cual el sistema, a diferencia de su entorno, se describe a sí mismo" (Luhmann 1991:395). Para el funcionalismo sistémico la reflexión es autoreferencia autopoiética, es decir, que elabora sus propios elementos mediante las operaciones de los mismos. En términos de la lógica de Hegel, como analizaremos en el próximo apartado, este tipo de autoreferencia sistémica es entendido como racionalidad. En ambos casos, en la reflexión de Luhmann y en la razón de Hegel, la unidad se produce a sí misma mediante sí misma.

\section{La racionalidad como auto-referencia}

Para Luhmann, como es sabido, la reflexión es la que opera en los diversos sistemas sociales de la sociedad moderna; lo que cierra la posibilidad de sustentar la idea de un único centro desde donde enjuiciar racionalmente. En el plano de la política, por ejemplo, "se producen desde el siglo XVII, teorías del Estado orientadas por el problema de que el mayor poder político tiene que ser capaz de superar a todas las 
fuerzas de un territorio y decidir cualquier conflicto, aunque deba abstenerse de un uso arbitrario. El resultado es la teoría del Estado Constitucional moderno" (Luhmann 1991:407). En el campo de la ciencia, "se producen teorías científicas que deben explicar la identidad en la diferencia entre conocimiento y objeto, ya sea como autodisciplinamiento de la conciencia trascendental, ya sea en forma de un proceso dialéctico, ya en forma de una pragmática abierta a la corroboración" (Luhmann 1991:407). Esta postura de racionalidad parcializada, que abandona la referencia exclusiva al sujeto o al espíritu, debe centrarse no en la unidad de unidad/diferencia -como Hegel- sino en la diferencia de unidad/diferencia. Dicho cambio de perspectiva es presentado por Luhmann como un instante de superación tanto del trascendentalismo kantiano (que reduce la auto-referencia al plano exclusivo del sujeto) como de la dialéctica hegeliana; caracterizando esta última como demasiado arriesgada: "Se llega al trascendentalismo cuando se comprende precisamente esto como característica especial de la conciencia y por ello se declara a la conciencia como sujeto. Se llega a la dialéctica cuando, ante este paralelismo de autorreferencia y referencia externa, se despierta interés por la unidad basal (en última instancia, basada en la identidad de identidad y diferencia, y no en la diferencia de identidad y diferencia). La dialéctica puede, aunque no necesariamente, combinarse con la teoría trascendental. Nosotros consideramos que la teoría trascendental es una manera falsa de convertir en absoluta sólo una referencia sistémica (pero también un buen modelo para teorías autorreferenciales), y que la dialéctica es demasiado arriesgada respecto de la identidad supuesta (siendo que las transiciones y los enlaces en la teoría deben partir siempre de la diferencia" (Luhmann 1991:399).

Habermas reconoce este aspecto: "ciertamente que la relación sistema-entorno es pensada punto por punto conforme al modelo de un mundo constituido por medio de la conciencia transcendental" (1993:446). La renovación funcionalista se basa en que "en lugar de un solo mundo fundado transcendentalmente, tenemos múltiples entornos relativos a sistema" (1993:446). Así, se "consigue con el concepto de sistema que elabora sentido una libertad de movimiento que le permite someter la sociedad como sistema social a un análisis parecido al de la conciencia como sistema psíquico" (1993:437).

Al igual que en la dialéctica de Hegel, en el funcionalismo sistémico la racionalidad se concibe como el nivel superior de auto-referencia. La tercera doctrina de la lógica, la doctrina del concepto, trata sobre "el espíritu vivo de lo efectivamente real" (Hegel 2005:248). Consta a su vez de tres sub-doctrinas. La primera, la del sujeto en cuanto tal, contiene las instancias de la universalidad (la igualdad consigo mismo), de la particularidad (el no ser otra cosa que uno mismo) y de la singularidad (reflexión hacia sí de las instancias anteriores), las que, según el autor, "son lo mismo que identidad, distinción y fundamento" (Hegel 2005:249). El determinar del concepto es el enjuiciar: "El juicio es el concepto en su particularidad, como referencia que distingue los momentos del concepto, los cuales están puestos como siendo [cada uno] de por sí y al mismo tiempo como idénticos [cada uno] consigo, no como idénticos los unos con los otros" (Hegel 2005:251). La unidad de concepto y juicio se manifiesta en el silogismo: el concepto en tanto unidad simple a la que han retornado las distinciones formales del juicio. De este modo: "Lo real efectivo es un singular que mediante la particularidad se eleva a la universalidad y se hace idéntico consigo mismo. Lo real efectivo es [algo] uno, pero es también el despliegue de los momentos del concepto, y el silogismo es el curso circular de la mediación de sus momentos por el cual lo efectivo se pone como uno" (Hegel 2005:251).

La segunda sub-doctrina del concepto se refiere al ser inmediato -indiferente a la distinción- que resulta de este proceso: el objeto. Este, mediante la negación de la exterioridad, se afirma como unidad negativa consigo misma o unidad autosuficiente. En cuanto a la totalidad del devenir del concepto, "es la contradicción entre esta totalidad suya y la determinidad de su existencia; por ello el objeto es la 
aspiración a superar esta contradicción e igualar su existencia al concepto" (Hegel 2005:276). Esta contradicción, que desgasta al uno mismo del objeto, la denomina Hegel astucia de la razón (2005:282). Durante este proceso, se desvanece "la oposición de contenido y forma. Al conectarse conclusivamente el fin consigo mismo mediante la superación de las determinaciones formales, la forma está puesta como idéntica consigo misma y por ende como contenido, de tal modo que el concepto sólo se tiene a sí mismo por contenido en cuanto actividad de la forma" (Hegel 2005:283).

El uno mismo de la idea, síntesis de las instancias anteriores, "es puro distinguir dentro de ella misma: intuir que se detiene en esta universalidad idéntica" (Hegel 2005:289). La filosofía "termina de este modo la comprensión del concepto de ella misma como concepto de la idea pura, para la cual la idea es" (Hegel 2005:300). Así, afirma Hegel: "La idea puede ser entendida como la razón (éste es el significado propiamente filosófico de razón); también como el objeto-sujeto, como la unidad de lo ideal y lo real, de lo finito y lo infinito, del alma y del cuerpo, como la posibilidad que tiene en sí misma su realidad efectiva, como aquello cuya naturaleza sólo puede ser concebida como existente, etc.; [y esto es así] porque en ella se contienen todas las relaciones del entendimiento, pero en su retorno infinito e identidad [vuelta] hacia sí" (2005:284).

De una manera similar, para Luhmann, la racionalidad se refiere a la distinción manifestada en la unidad de la distinción: "La racionalidad sólo se da cuando el concepto de diferencia se utiliza de modo autorreferencial, es decir, cuando se refleja en la unidad de la diferencia" (1991:420). Esto quiere decir que, para cada sistema social, que "ellos mismos se determinan a sí mismos diferenciándose respecto del entorno y deben otorgar a esta diferencia un significado operativo, un valor informativo, un valor de enlace" (Luhmann 1991:420). Ahora bien, como hemos visto, la racionalidad no se centra en la manifestación de un todo que no posee referencia proveniente del exterior como el espíritu en Hegel, sino en los diversos sistemas sociales. Al menos esto pretendía Luhmann: "La lógica del observar y describir debe reajustarse de estructuras monocontexturales a estructuras policontexturales" (2007:896). Así, las operaciones de cada sistema "se vuelven objeto posible de otra distinción, la cual expone su propio tertium non datur a que otros observadores lo aprehendan" (2007:896). La ciencia no puede entonces exigir la validez última o modificar las operaciones de los otros sistemas. Cada sistema es entorno de todos los demás.

Al comienzo de La sociedad de la sociedad el autor sostiene que la idea de forma de la teoría de sistemas tiene similitudes con el término hegeliano de concepto: "Este concepto de forma tiene cierta semejanza con el término hegeliano de concepto en cuanto que para ambos es constitutivo incluir una distinción" (Luhmann 2007:41). No obstante, en Hegel el concepto -en tanto existencia autosuficiente- es una unidad libre: es capaz de "determinar su ritmo por sí mismo" (Hegel 2011:38). En Luhmann, al contrario, la unidad no puede realizar su propia unidad mediante sí misma. La unidad de la forma es más bien un tercer excluido que no puede observar la observación. Mientras que en Hegel "el concepto se preocupa por resolver por sí mismo el problema de su unidad con lo cual elimina la autonomía de lo distinto: por ejemplo, la autonomía de los momentos contrapuestos de capacidad sensorial y razón, en el concepto de hombre" (Luhmann 2007:41), en Luhmann, "la forma es precisamente la distinción misma en cuanto que apremia a señalar (y por tanto a observar) un lado o el otro y por esa razón no puede ella misma (muy distinto al concepto hegeliano) realizar su propia unidad. La unidad de la forma no es su más alto sentido espiritual" (2007:41); la postura de Hegel, al contrario de la de Luhmann, es propia de una unidad pensada como totalidad no observada sino por sí misma. No obstante, "la conceptualización del cálculo de la forma de Spencer Brown presupone el tiempo, trabaja con el tiempo, se explicita a sí misma con él, al igual que la lógica de Hegel" (Luhmann 1998:55). 
Para Luhmann, cuando el problema de las condiciones incondicionadas de la razón, constante en todo el pensamiento tradicional europeo, se plantea como pretensión de un sistema parcial de la sociedad, la respuesta debe ser distinta: "en el ámbito de su observar distinguía y señalaba, pero ahora es a ella misma [la filosofía] a quien se distingue y se señala" (2007:899). Si la auto-referencia opera dentro de una sociedad poli-funcional, no puede "evitarse el concebir todo observar y describir como ocultamiento y despliegue de la paradoja de la unidad; se entiende de por sí que eso puede suceder de múltiples maneras" (2007:899). Por este motivo: "a more general theory of profiting from the development of sociological systems on the one hand, and answering the search for a social theory beyond rational modern society on the other, is a theory of complex systems whose starting point is the observer [...] Yet and soon as an observer among other observer of a similar or different kind enters a relationship of second-order observation, this relationships should go by a name to be delineated, accessed, left again, and distinguished from other relationships" (Baecker 2014:29).

Sin embargo, a pesar de esta diferencia, sigue existiendo un paralelo considerable: de la misma manera que en Hegel el espíritu, mediante la filosofía, se sabe a sí mismo como origen y destino de todo, es solamente la sociedad la que "se observa a sí misma como observador, se describe a sí misma como descriptor y sólo esto es justo autodescripción o bien autoobservación en un sentido estrictamente lógico. Apenas ahora lo auto de la observación es el observador, lo auto de la descripción es el descriptor mismo" (Luhmann 2007:904). Tanto la sociedad del funcionalismo como el espíritu de la dialéctica hegeliana son auto-referencia indeterminada, a pesar de que Luhmann haya pretendido formular una dialéctica sin espíritu: "la sociedad es un sistema que se observa y describe así mismo" (1998:58). La lógica de la producción como auto-referencia inevitablemente debe arribar en la idea de una determinación indeterminada, de un sujeto universal.

Este enunciado se ha confrontado - desde inicios del siglo XIX-con la idea kantiana del despliegue histórico de las propiedades que al ser humano le son inherente (al que Habermas se refiere como promesa de la razón): "For Kant, in keeping with his subordination of historical considerations to practical reason and its moral mandate, philosophy of history was about a project. He organized the problem of history into a series of tasks or phases of that project, and hence history had a clear telos. The task was to realize an ethical community in this world, to establish the "Kingdom of God» on earth. Within that moral project, there was a distinct political project: the establishment of «republican constitutions» within individual communities and, as the necessary environment for their viability, of a "perpetual peace» among states governed by a similar respect for universal right. For Hegel, history is ultimately about an insight: the recognition that history is the achievement -literally the self-actualization- of human freedom, by the force of reason. The actualization of freedom is, to be sure, also a matter of institutionalization: Hegel shares Kant's concern for political organization, though their models differ widely. But the telos of history, from the vantage of philosophy of history, is a matter of self-recognition of reason as constitutive of the (historical) world" (Zammito 2012:838).

A estas dos caras se le ha denominado humanismo positivo y humanismo negativo, lo que permite hablar del "negativamente humanista antihumanismo teórico de Niklas Luhmann" (Miranda 2012:279).

Por los motivos anteriormente mencionados no es casualidad que, en el plano normativo, el phatos del funcionalismo sistémico sea tan congruente con el de los hegelianos de derecha del siglo XIX: "no cabe duda de que precisamente en este pathos de Luhmann, en este su sentido de la realidad aliado con las racionalidades parciales institucionalizadas, nos topamos con una herencia muy alemana, persistente desde los hegelianos de derechas devenidos escépticos hasta Gehlen" (Habermas 1993:440). En ambos 
casos se habla del devenir de una totalidad incondicionada, que impide justificar posturas normativas de transformación: "la exposición del problema de la racionalidad no significa que la sociedad tenga que resolver problemas de este tipo para asegurar su supervivencia; para sobrevivir basta la evolución" (Luhmann 1991:423).

Acusaciones similares que los hegelianos de derecha devenidos escépticos realizaron en contra del liberalismo o el socialismo, son efectuadas por el funcionalismo sistémico en contra de la teoría crítica o la metafísica de occidente. Para los primeros, "la sociedad aparece a nativitate como una esfera de la desigualdad de necesidades naturales, de caudal y de habilidades; constituye un plexo objetivo cuyos imperativos funcionales inevitablemente penetran a través de las orientaciones subjetivas de la acción" (Habermas 1993:92), por la que cualquier intento de establecer principios normativos universales resulta invalido. Para Luhmann, de la misma manera, en una sociedad poli-funcional no existe un centro que permita enunciar principios universales. En la sociedad funcionalmente diferenciada la racionalidad aparece como universo fragmentado, en donde cada sistema presenta pretensiones de universalidad. El punto de los hegelianos de derecha es renovado pero no como orientaciones subjetivas de acción sino como sistemas independientes de comunicación (sin considerar al Estado como centro): "todos los sistemas funcionales reclaman universalidad -aunque tan solo para su ámbito correspondiente" (Luhmann 2007:779); por tanto, "la lógica del observar y describir debe reajustarse de estructuras monocontexturales a estructuras policontexturales" (Luhmann 2007:896).

Para el caso de la valorización del saber existen paralelos similares. En los hegelianos de derecha, las ciencias del espíritu eran las encargadas de reemplazar la herencia de una filosofía muerta o de un Estado envenenado por la burocratización: "quien entable pretensiones teoréticas de más alto alcance, quien siguiendo los pasos de los «maitres penseurs» siga cultivando la filosofía y la teoría de la sociedad, se delata a sí mismo como intelectual -un seductor so capa de ilustrador, que participa del afán de sacerdotal dominación que mueve a la nueva clase" (Habermas 1993:97). De la misma forma, en Luhmann, la labor de la teoría de sistemas sociales "no es continuar los esfuerzos asombrosos realizados por la filosofía ni exaltar la utilidad de sus resultados [...] La sociología no tiene ciertamente las posibilidades tan ricas de formulación literaria con las cuales la filosofía puede presentarse. Debe poner atención a la cientificidad, lo cual no por último es cuestión de estilo" (2007:736). El funcionalismo se auto-considera como mecanismo de observación compleja de la sociedad, como el resultado (la teoría) del resultado (diferenciación funcional), ajeno a las pretensiones universales de la vieja Europa: "digamos que esta teoría de sistemas no pone a la sociología en el camino más seguro de una ciencia, sino que más bien se presenta como sucesora de una filosofía a la que se supone cancelada" (Habermas 1993:434).

Ahora bien, es importante reconocer que si bien en los puntos anteriores Luhmann coincide con los hegelianos de derecha, en lo que se refiere al valor de la tradición la situación es distinta. Los hegelianos de derecha consideran que sin la capacidad de integración que ofrece la tradición, no habría obstáculo frente a los peligros de la modernidad: "de aquí parte la idea historicista de que la libertad subjetiva, que aparece en el modo de la disociación y escisión, sólo puede defenderse contra los peligros de una socialización y burocratización totales si los devaluados poderes de la tradición asumen, no obstante, un poder compensatorio" (Habermas 1993:95). De forma contraria, Luhmann sostiene que "cuando decimos que sólo las comunicaciones y todas las comunicaciones contribuyen a la autopoiésis de la sociedad y que con eso la característica de omniabarcador se redefine, está contenida en esa tesis también una amplia ruptura con la tradición" (2007:64). Así, "todos los supuestos sobre el acuerdo, el progreso, la racionalidad y otros fines bien vistos serán remitidos a una posición teórica secundaria" (2007:65). 


\section{La concordancia entre teoría y sociedad}

Existe otro cuarto aspecto en el que es posible relacionar la obra de Luhmann con la de Hegel: en ambos casos, el pensamiento o la teoría -debido a que forma parte de lo descrito-adquiere la misma modalidad que la realidad histórica a la que se refiere.

En Hegel las consideraciones anteriores sobre el universo metafísico son concebidas como momentos históricos en la progresiva auto-consciencia del espíritu; no como errores lógicos: "Estas manifestaciones del pensamiento, en las que éste se encuentra a sí mismo, son las filosofías; y la cadena de estos descubrimientos, de los que parte el pensamiento a descubrirse a sí mismo, es la obra de tres mil quinientos años" (1995:11). El pensamiento racional "no ha surgido de improvisto, directamente, como si brotase por sí sola del suelo del presente, sino que es también, sustancialmente, una herencia y, más concretamente, el resultado del trabajo de todas las anteriores generaciones del linaje humano" (1995:9). El propósito de la reflexión filosófica no se reduce a "a exponer el aspecto externo, lo ocurrido, los acaecimientos que se refieren al contenido, sino en hacer ver cómo el contenido -en cuanto se manifiesta históricamente- forma parte de la misma ciencia de la filosofía" (1995:13). En el desarrollo del espíritu de la misma manera que el devenir desde la conciencia hasta la autoconsciencia- cada fase corresponde a la unidad de unidad/diferencia que retorna a sí misma: "como el embrión en la naturaleza, también el espíritu, desde de haberse hecho otro, retorna a su unidad; pero lo que es en sí deviene para el espíritu y deviene, por consiguiente, para sí mismo" (1995:27). El contenido de la evolución en la historia humana es, por ende, lo concreto o la unidad de lo distinto: "lo concreto es, por tanto, simple y al mismo tiempo, a pesar de ello, distinto. Esta contradicción interna de lo concreto, que es precisamente la que sirve de acicate a la evolución, da origen a las diferencias" (1995:29). De esta manera, por ejemplo, "la filosofía jonia surge al sobrevenir la decadencia de los Estados jónicos en el Asia menor" (1995:54), o "en Roma, la filosofía no empieza a difundirse hasta que no se hunde la auténtica vida romana, la de la República" (1995:54). En síntesis, "la filosofía sólo aparece al llegar una determinada época en la formación del todo" (1995:55).

En la obra de Luhmann, esta perspectiva aparece bajo la forma de teoría de la evolución. Al igual que en Hegel la filosofía corresponde a un instante de la historia del espíritu, en Luhmann, las diversas autodescripciones de la sociedad "presentan marcadas similitudes estructurales respecto a la tipología de su diferenciación" (2007:708). A pesar de que Luhmann haya pretendido elaborar una teoría sin espíritu, el rol que este cumple en la filosofía de Hegel (en términos de autorrealización) en la teoría social de sistemas es otorgado, como vimos anteriormente, a la sociedad total (en términos de diferenciación interna o de supra-sujeto): en ambas instancias no existe referencia proveniente del exterior. Por este motivo (la filosofía como reflejo de la auto-conciencia del espíritu, la semántica como reflejo de la diferenciación de la sociedad), tanto en Hegel como en Luhmann el pensamiento opera de forma similar a la realidad observada.

Por ejemplo, en las sociedades tribales, según Luhmann, debido al escaso nivel de diferenciación, toda observación del mundo inicia con la idea de la presencia de un continuo de realidad, donde las distinciones entre entes se entienden como diferencias jerárquicas entre esencias organizadas cósmicamente. El mundo social se ordena en géneros/especies bajo el supuesto de una lógica todo/partes: "Tanto las divisiones centro/periferia como también los órdenes jerárquicos señalan posiciones en el centro o en la cima desde las cuales puede describirse, sin competencia, al mundo y a la sociedad" (2007:723). En la sociedad moderna, de la misma manera, "en la medida en que la diferenciación de los sistemas funcionales adquiere preminencia, cambia también la onticidad de los objetos, la exclusividad de su ser y lo adecuado 
de orientarse hacia ellos en el conocer y el actuar" (2007:751). La transformación desde la ontología hacia el funcionalismo de Luhmann "es correlativo a la transformación del orden social estratificado tradicional en otro funcionalmente diferenciado, y que sólo ahora es claramente observable en sus principales rasgos y consecuencias" (Beriain y García 1998:15).

El funcionalismo se considera consecuencia de una sociedad en la que ya no es posible una "observación externa adecuada" (Luhmann 2007:694). En vez de la intersubjetividad "ahora aparece el hecho de que las autoobservaciones y las auto-descripciones sociales se exponen a su vez a la observación y a la descripción -dado que solo pueden ocurrir como comunicación" (Luhmann 2007:695). Por tanto, "la sociedad debe entonces describirse con metaconceptos -como policontextural o hipercompleja. Entonces cada autodescripcion, en cuanto descripción, toma en cuenta su propia contingencia. Considera (y hace ver que considera), que puede haber también otras autodescripciones del mismo sistema" (Luhmann 2007:697). En síntesis: se piensa funcionalmente debido a que la realidad misma se compone de diversos sistemas funcionales: "si la sociedad moderna puede ser descrita como un sistema social funcionalmente diferenciado, entonces nos encontramos ante una sociedad caracterizada tanto por la desigualdad como por la simetría en las relaciones entre sus sistemas parciales" (Beriain y García 1998:15). Por esto, como adelantamos en la introducción, no es casualidad que los argumentos de Luhmann-y de los adherentes a la teoría de Luhmann- para justificar la aplicación de los postulados de la teoría de sistemas o la fenomenología a la teoría de la sociedad no sean teóricos, sino históricos: es la realidad de la sociedad la que ya no permite referirse a esta con los postulados de la sociología tradicional. No es casualidad, tampoco, que al igual que el panorama de los herederos de Luhmann sea similar a los herederos de Hegel. Como sostiene Sloterdijk, Luhmann "use the final possibilities of a given grammar to the full, and thus give their successors the initially euphoric feeling of starting at a high point. This subsequently gives way to the alarming realization that if one starts at the peak, the only way to continue is downwards" (2009:3).

Ahora bien, es necesario considerar que si bien para ambos autores el conocimiento constituye una instancia de superación, en Hegel este posee un destino ya previsto: "quien en los acontecimientos que se producen en el campo del espíritu, las filosofías, sólo vea contingencias, no toma en serio la fe en un gobierno divino del universo" (Hegel 1995:36); "lo contingente debe ser abandonado a la puerta misma de la filosofía" (Hegel 1995:40). En Luhmann, en cambio, en el contexto de una sociedad funcionalmente diferenciada ya no es posible referirse a un propósito que trascienda el devenir de los acontecimientos humanos: "La relación entre la teoría de la evolución y la dialéctica (y, por tanto, con la teoría de la historia de Hegel) exigiría una investigación más profunda. Nótese aquí únicamente que el concepto de forma marca una distinción y, por consiguiente, presenta como necesario el enlace entre ambas partes de la distinción. Por eso, a la variación sigue necesariamente la selección; a la selección sigue necesariamente la reestabilizacion. Sin embargo, esto no significa que sea necesario el proceso correspondiente. Ni significa tampoco que dentro de este proceso se active el movimiento únicamente como consecuencia de las distinciones que se constituyen como oposiciones. Estas premisas pueden mantenerse solo si se postula algo como espíritu" (Luhmann 2007:394).

Aunque, como analizamos anteriormente, si bien la idea de espíritu es ajena a la teoría social de sistemas, en lo que se refiere a las consecuencias normativas de la teoría, la idea de sociedad como supra-sujeto cumple la misma labor que la idea de espíritu tenía en Hegel. 


\section{Consideraciones finales}

En la sociedad occidental pre-moderna, la historia del mundo cristiano estaba compuesta por un conjunto de profecías oficiales referidas al retorno de Cristo. En cada una de estas, participaban figuras arquetípicas universales para representar el conflicto entre el bien y el mal. Los asuntos humanos eran concebidos como símbolos de una estructura no-histórica del devenir. No se formaba todavía la concepción moderna del tiempo como proceder lineal, irrepetible e irreversible. Todos los sucesos eran interpretados en torno a las variables de la lucha entre el Cristo y el Anticristo o como una nueva manifestación de un proceder profético.

Desde el siglo XVI las fechas estimadas para el retorno de Cristo comienzan continuamente a ser aplazadas. La última profecía papal de 1595 -sustentada en las visiones de San Malaquías- aseguraba el inicio del Apocalipsis a principios de 1990. Con el fin de la perspectiva profética del devenir aparece el futuro como un campo de posibilidades indeterminadas. Lo que anteriormente era profecías, en el pensamiento ilustrado es pronóstico: designio racional. En la especulación escatológica el hecho es un símbolo: representa la forma de toda la historia. En el pensamiento moderno, en cambio, el hecho es consecuencia de un evento anterior.

La transformación que inaugura la conciencia moderna de la historia fue precisamente la filosofía de la historia, cuyas manifestaciones principales fueron las obras de Kant y Hegel. Como indica Foucault, al igual que en la psicología, el surgimiento del saber con el del objeto-conocido es paralelo. La filosofía de la historia se origina como una particular mezcla entre política y profecía: una combinación entre pronóstico racional ilustrado y la esperanza de salvación propia del dogma cristiano. Lo que antes era potencialidad incierta, con la filosofía de la historia es futuro determinado: utopía. El plano de la inmanencia se subordina al de la trascendencia, dirían Hardt y Negri.

Las teorías de la sociedad de las ciencias sociales, en donde -como hemos expuesto en este artículo por medio del caso de Luhmann- las posturas de la filosofía de la historia experimentan un proceso de secularización, son parte de esta misma evolución. La teoría de la sociedad es a la filosofía de la historia del siglo XIX, lo que esta fue para la escatología medieval, a saber, un instante de transformación en donde las instancias anteriores perviven, a la vez que la conciencia histórica se radicaliza cada vez más.

\section{Bibliografía}

Baecker, D. 2014. Complex systems in social theory. Social Science Research Network. doi: $10.2139 /$ ssrn. 2512647

Beriain, J. y García, J. 1998. Introducción. En: N. Luhmann. Complejidad y modernidad. De la unidad a la diferencia. Madrid: Trotta, pp. 9-24.

Casanova, M. 2014. Razón y normatividad: el debate entre Jürgen Habermas y Niklas Luhmann. Tesis para optar al grado de Magíster en Filosofía. Santiago: Universidad de Chile.

Enthoven, J. 2009. Auguste Comte y el positivismo. En: Y. Belaval. La filosofía en el siglo XIX. México: Siglo XXI, pp. 198-278.

Gadamer, H. 1993. El problema de la conciencia histórica. Madrid: Tecnos.

Habermas, J. 1993. El discurso filosófico de la modernidad: Madrid: Trota. 
Habermas, J. 2000. Conciencia moral y acción comunicativa. Barcelona: Península.

Habermas, J. 2000a. Aclaraciones a la ética del discurso. Madrid: Trota.

Habermas, J. 2003. Teoría de la acción comunicativa II: crítica de la razón funcionalista. Madrid: Taurus.

Hegel, G. W. F. 1955. Lecciones sobre la historia de la filosofía. México: Fondo de Cultura Económica.

Hegel, G. W. F. 1966. Fenomenología del espíritu. Madrid: Fondo de Cultura Económica.

Hegel, G. W. F. 2005. Enciclopedia de las ciencias filosóficas. Madrid: Alianza Editorial.

Hegel, G. W. F. 2011. Ciencia de la lógica. México: Abada Editores.

Henrich, D. 2002. Between Kant and Hegel: lectures on German idealism. London: Harvard University Press.

Izuzquiza, I. 2008. La sociedad sin hombres: Niklas Luhmann o la teoría como escándalo. Barcelona: Anthropos.

Koselleck, R. 1993. Futuro pasado: para una semántica de los tiempos históricos. Barcelona: Paidós.

Larraín, J. 2007. Comentario a sociología de la cultura: la deconstrucción de lo mapuche de Aldo Mascareño. Estudios públicos 105: 113-120.

Luhmann, N. 1991. Sistemas sociales: lineamientos para una teoría general. México: Alianza

Luhmann, N. 1996. La ciencia de la sociedad. México: Anthropos.

Luhmann, N. 1998. Complejidad y modernidad. De la unidad a la diferencia. Madrid: Trotta.

Luhmann, N. 2007. La sociedad de la sociedad. México: Herder.

Lyotard, J. 2006. La condición posmoderna. Madrid: Cátedra.

Mascareño, A. 2009. Medios simbólicamente generalizados y el problema de la emergencia. Cinta de Moebio 36: 174-197. doi: 10.4067/S0717-554X2009000300003

Miranda, P. 2012. La pre-comprensión de lo humano en la sociología de Luhmann. Raíces antropológicas del antihumanismo teórico luhmanniano. Santiago: Ediciones Universidad Alberto Hurtado.

Perinetti, D. 2006. Philosophical reflexion on history. En: The Cambridge history of eighteenth-century philosophy. Cambridge: Cambridge University Press, pp. 1107-1140.

Pippin, R. 2012. The kantian aftermath: reaction and revolution in German philosophy. The Cambridge history of philosophy in the nineteenth century (1790-1870). Cambridge: Cambridge University Press, pp. 19-45.

Rodríguez, D. y Arnold, M. 2007. Sociedad y teoría de sistemas. Santiago: Universitaria.

Sloterdijk, P. 2009. Derrida, an Egyptian. On the problem of the Jewish pyramid. London: Polity Press.

Zammito, J. 2012. Philosophy of history: the German tradition from Herder to Marx. The Cambridge history of philosophy in the nineteenth century (1790-1870). Cambridge: Cambridge University Press, pp. 817-865.

Recibido el 10 Oct 2015

Aceptado el 17 Dic 2015 